\title{
Panaxdiol saponins component promotes hematopoiesis by regulating GATA transcription factors of intracellular signaling pathway in mouse bone marrow
}

\author{
Tie-Ying Dai ${ }^{1}$, Jin-Jian Lan ${ }^{1}$, Rui-Lan Gao ${ }^{1}$, Yan-Na Zhao ${ }^{1}$, Xiao-Ling Yu ${ }^{1}$, Simon-Xun Liang ${ }^{1}$, \\ Wen-Bin Liu ${ }^{1}$, Xin Sun ${ }^{2}$ \\ ${ }^{1}$ Institute of Hematology Research, The First Affiliated Hospital of Zhejiang Chinese Medical University, Hangzhou, China; ${ }^{2}$ Department of \\ Oncology, Zhejiang Provincial People's Hospital, Hangzhou, China \\ Contributions: (I) Conception and design: X Sun, TY Dai; (II) Administrative support: SX Liang, RL Gao; (III) Provision of study materials or \\ patients: YN Zhao, XL Yu; (IV) Collection and assembly of data: JJ Lan; (V) Data analysis and interpretation: TY Dai, WB Liu; (VI) Manuscript \\ writing: All authors; (VII) Final approval of manuscript: All authors. \\ Correspondence to: Xin Sun. Department of Oncology, Zhejiang Provincial People's Hospital, No. 158 Shangtang Road, Hangzhou 310014, China. \\ Email: sunxin842021@163.com.
}

Background: Our research team has identified a biological active component, panaxadiol saponins component (PDS-C) isolated from total saponins of panax ginseng as a potential targeted drug for treating hemocytopenia. PDS-C possesses dual activities, namely that of promoting hematopoiesis and regulating immune function. Our study is to observe effects of PDS-C on promoting hematopoiesis in normal and aplastic anemia (AA) mice, furthermore, to explore its possible mechanism.

Methods: Bone marrow nucleated cells were cultured for colony forming assay of CFU-GM, CFU-E and CFU-MK in the presence of PDS-C at different concentration. The proliferation and differentiation-related genes expression profile was analyzed with DNA membrane microarray. The mRNA expression levels and protein phosphorylated state of GATA-1, GATA-2 transcription factors and AKT-1, MAPK14 protein kinases were detected by RT-qPCR and Western blot, the DNA binding activity and components of GATA-DNA complex were analyzed by EMSA and antibody gel supershift assay.

Results: In response to PDS-C at 10, 25 and $50 \mathrm{mg} / \mathrm{L}$, the bone marrow colony numbers of CFU-GM, CFU-E and CFUMK increased significantly by $25.7 \% \pm 3.1 \%$ to $42.4 \% \pm 4.5 \%$ respectively in normal mice, and $29.7 \% \pm 3.7 \%$ to $53.2 \% \pm 7.1 \%$ in AA mice. The gene microarray profile initiated by PDS-C provided the up-regulated genes by more than 3 times, which can be classified into 11 categories according to their functions, including GATA-1, GATA-2, and AKT-1, MAPK14. The mRNA expression levels of GATA-1, GATA-2 were consistent with their gene microarray profile in PDS-C treated erythroid and megakaryocytic hematopoietic cells. Meanwhile, PDS-C could not only up-regulate expression levels of GATA-1, GATA-2 proteins, but also enhance phosphorylated activity state. Furthermore, PDS-C obviously enhanced binding activity of GATA protein with DNA in erythroid and megakaryocytic cells, and the main composition of GATA-DNA complex was GATA-2 and GATA-1.

Conclusions: PDS-C displays the role to promote proliferation and induce differentiation for hematopoietic cells. Its action mechanism may involve in GATA-1, GATA-2 transcription factors, including up-regulating mRNA and protein expression, enhancing DNA binding activity, phosphorylated functional activity and up-regulating AKT-1, MAPK14 protein kinases as the upstream signaling molecule for activation GATA-1, GATA-2 respectively in hematopoietic cells.

Keywords: Panaxadiol saponins component (PDS-C); hematopoietic cells; GATA transcription factor; protein kinases

Submitted Aug 17, 2021. Accepted for publication Dec 24, 2021.

doi: $10.21037 / \mathrm{atm}-21-4800$

View this article at: https://dx.doi.org/10.21037/atm-21-4800 


\section{Introduction}

At present, hemocytopenias such as aplastic anemia (AA), primary immune thrombocytopenia (ITP), and chronic neutropenia (agnogenic leukocytopenia) are routinely treated by androgen, glucocorticoids, and immunosuppressive agents (1-3). However, the efficacy of these treatments is variable and uncertain. In addition, they frequently cause serious side effects. There are several Chinese patent medicines used in treating hematocytopenia, but their effectiveness is unsatisfactory. Therefore, there is an urgent need to study and develop safe and effective new Chinese patent medicines for treating hemocytopenia.

Our research team has identified a biological active component from the Chinese ginseng herb. Panaxadiol saponins component (PDS-C) was isolated from total saponins of panax ginseng and formulated into capsules named Pai-neng-da. The composition and content of PDS-C was identified by high performance liquid chromatography-mass spectrometry (HPLC-MS) using specific ginsenoside monomers as reference standards. We also performed preclinical studies of PDS-C as these were required for the patent of class-five new Chinese medicine (4-7). Finally, we successfully obtained approval from the China Food and Drug Administration (CFDA) to conduct clinical trials of PDS-C and its derived Pai-neng-da capsule as a new Chinese patent medicine.

Our previous studies of animal model studies, cellular, and molecular biology experiments have demonstrated that PDS-C possesses dual activities, namely that of promoting the proliferation and differentiation of hematopoietic progenitor cells, and that of regulating the immune function (7-9). PDS-C was shown to enhance proliferation and differentiation of granulocytic, erythroid and megakaryocytic progenitor cells (CFU-GM, CFU-E, CFU-MK) of human bone marrow, it revealed growth factor-like action and synergistic effects with growth factors $(8,9)$. In view of the efficacy mechanism of PDS-C is not yet clear, in this study, the hematopoietic progenitor cells of mouse bone marrow were treated by PDS-C, then, their proliferation and differentiation-related genes expression profile was analyzed by DNA membrane microarray. The messenger RNA (mRNA) expression levels and protein phosphorylated state of GATA-1 and GATA-2 transcription factors were detected by quantitative reverse transcription polymerase chain reaction (RT-qPCR) and western blot, DNA binding activity and components of GATA-DNA complex were analyzed by electrophoretic mobility shift assay (EMSA) and antibody gel supershift assay. Since GATA-1 is participated in the development of erythrocyte and differentiation of megakaryocyte, while, GATA-2 are involved in the proliferation of early hematopoietic cells. Meanwhile, protein kinases of AKT-1 and MAPK14 were tested by western blot, so as to explore the intracellular signal pathway induced by PDS-C in hematopoietic cells. We present the following article in accordance with the ARRIVE reporting checklist (available at https://atm. amegroups.com/article/view/10.21037/atm-21-4800/rc).

\section{Methods}

\section{Dry fine powder of PDS-C}

The effective components of PDS-C were isolated from total saponins of panax ginseng by macroporous resin at the First Affiliated Hospital of Zhejiang Chinese Medical University. The composition of PDS-C was analyzed and determined by HPLC, using specific monomers of ginsenoside as reference standards (7-9). The dry fine powder of PDS-C was completely dissolved in distilled water, and filtrated for sterilization.

\section{Bone marrow nucleated cells from normal and AA mouse}

We purchased BALB/c mice from the Shanghai Si-LaiKe Experimental Animals Inc. in China (SCXK 20120002, specific pathogen free grade). Animals were cared for in accordance with the guidelines of the National Science and Technology Committee of China. All procedures and animal experiments were approved by the Animal Care and Use Committee of the Animal Center of Zhejiang Chinese Medical University (No. ZSLL-2015-126). A protocol was prepared before the study without registration. The male and female BALB/c mice, weighing 20-22 g, 3-4-week-old, were used for the following experiment. The nucleated cell suspension samples of bone marrow were acquired from mouse femurs for colony forming assay.

The AA mouse model was established as previously described (10). Briefly, BALB/c mice were exposed to 5.0 Gy total body irradiation (TBI) at approximately $1.0 \mathrm{~Gy} / \mathrm{min}$, and injected via tail vein with a single dose of $2 \times 10^{6}$ lymphocytes isolated from lymph glands of DBA mice within $4 \mathrm{~h}$ after irradiation. On day 7 after establishment of AA model, the mice were sacrificed by cervical dislocation, and nucleated cells were obtained from AA mouse femurs for colony forming assay of bone marrow culture. 


\section{Colony formation assay of CFU-GM, CFU-E, and CFU-MK}

The bone marrow nucleated cells were obtained from the femur of both normal mice and AA mice, and plated onto wells of tissue culture plates using a semisolid culture system for CFU-GM and CFU-E culture, in Iscove's modified Dulbecco's medium (IMDM) consisting of $20 \%$ fetal bovine serum (FBS), $10 \mu \mathrm{g} / \mathrm{L}$ recombinant murine granulocyte macrophage-colony stimulating factor or erythropoietin (GM-CSF or Epo, Peprotech, Rehovot, Israel) respectively, and $0.3 \%$ agar as viscous support with $10^{5}$ nucleated cells per well, incubated at $37^{\circ} \mathrm{C}$ with $5 \% \mathrm{CO}_{2}$. The colony numbers of CFU-GM ( $\geq 40$ cells) and CFU-E ( $\geq 8$ cells) were counted after culture for 5 days $(11,12)$. For CFU-MK culture, the nucleated cells were cultured in IMDM consisting of $20 \% \mathrm{FBS}, 1 \%$ bovine serum albumin, $10^{-5} \mathrm{~mol} / \mathrm{L}$ 2 -mercaptoethanl, and $10 \mu \mathrm{g} / \mathrm{L}$ recombinant murine thrombopoietin (Tpo, Peprotech, Israel), and incubated for 7 days. Murine megakaryocytes within CFU-MK colonies were identified by acetylcholinesterase staining as previously described (9), and the colony numbers of CFU$\mathrm{Mk}$ ( $\geq 4$ cells) were counted after culture for 7 days.

All experiments were performed in triplicate with $10^{5}$ nucleated cells per well. The solution of PDS-C was diluted and added to culture system of CFU-GM, CFU-E, and CFU-MK at final concentrations of $0,5,10,25,50$, and $100 \mathrm{mg} / \mathrm{L}$ respectively, and the increasing rates of colony formation induced by PDS-C were calculated after bone marrow culture. The positive control agent was Testosterone of androgen (Sigma, USA) at final concentration of $10^{-7} \mathrm{M}$ in the culture system. As androgens that promote the proliferation of hematopoietic cells, is commonly used to treat AA in China.

\section{Gene expression profile with membrane microarray}

The mouse bone marrow culture of CFU-GM, CFU-E, and CFU-MK was as same as above, but using methylcellulose instead of agar as viscous support, and treated by PDS-C $50 \mathrm{mg} / \mathrm{L}$ for 3 days respectively. The myeloid, erythroid, and megakaryocytic hematopoietic cells were harvested after washing away of methylcellulose. Total RNA was extracted from PDS-C treated cells and control cells, Poly+(A) mRNA was isolated from total RNA using Oligotex mRNA MIDI kit (Qiagen, Valencia, CA, USA), which was retrograded into complementary DNA (cDNA) and labeled with $\left[\alpha-{ }^{33} \mathrm{P}\right]$ dATP [Amersham (GE Healthcare),
Wauwatosa, WI, USA] as the probe.

The gene membrane microarray of $480 \mathrm{cDNA}$ target genes were provided and prepared by Shanghai Zhongke Kairui Biochip Technology Co., Ltd. (Shanghai, China). All target genes were related to cellular proliferation and differentiation with about 1,000 bp in length, dissolved in 3 times saline sodium citrate (SSC) solution at $0.5 \mu \mathrm{g} / \mu \mathrm{L}$, and sampled on $8 \mathrm{~cm} \times 12 \mathrm{~cm}$ nylon membrane with NOBG600 sampling point instrument (PROCEPT BioRobotics, Redwood Shore, CA, USA). The prepared gene membrane microarray was put into the hybrid tube, $4 \mathrm{~mL}$ of hybrid solution and $40 \mu \mathrm{L}$ of denatured fish sperm DNA $(100 \mu \mathrm{g} / \mathrm{mL})$ were added, and $40 \mu \mathrm{L}$ of $\left[\alpha-{ }^{33} \mathrm{P}\right]$ labeled probe was added, then, it was placed into the hybrid furnace at $68^{\circ} \mathrm{C}$ for $24 \mathrm{~h}$. The hybrid membrane microarray was placed in the lotion, and after repeated washing, the membrane microarray was scanned with BAS-MS 2340 phosphorous screen scanner (Fujifilm, Tokyo, Japan). The scan data of signal grayscale were taken and analyzed as the quantification value of signals intensity and ratio by ImaGene software (https://www.imagenelabs.com/), which are the intensity and ratio of signals.

\section{Nuclear extracts and total cell lysate}

The method of cells culture and PDS-C treatment were as same as gene membrane microarray. The nuclear extracts were prepared for EMSA as in the authors' previous study $(13,14)$, the cells were suspended in hypotonic buffer A $(10 \mu \mathrm{g} / \mathrm{mL}$ of the mixture of aprotinin, leupeptin, antipain, and pepstatin) for $15 \mathrm{~min}$. Subsequently, 0.6\% Nonidet P-40 was added and vortexed. Nuclei were separated from the cytosol by centrifugation and re-suspended in hypotonic buffer C (20 mM [4-(2-hydroxyethyl)-1piperazineethanesulfonic acid) (HEPES), 25\% glycerol, $0.4 \mathrm{mM} \mathrm{NaCl}, 1 \mathrm{mM}$ ethylenediamine tetracetic acid (EDTA), $1 \mathrm{mM}$ DL-Dithiothreitol, $0.5 \mathrm{mM}$ phenylmethanesulfonyl fluoride (PMSF), and $10 \mu \mathrm{g} / \mathrm{mL}$ of protease inhibitors mixture] and briefly sonicated on ice. Nuclear extracts were obtained after centrifugation. Total cell lysate was then prepared for western blot analysis, radioimmunoprecipitation assay (RIPA) buffer [1\% Nonidet P-40, 0.5\% sodium deoxycholate, $0.1 \%$ sodium dodecyl sulfate (SDS)] was added, followed by freshly prepared protease inhibitors mixture, and briefly sonicated on ice. The total cell lysate was obtained after centrifugation. 


\section{RT-qPCR for mRNA of GATA transcription factor}

Total RNA was extracted from cultured cells using Trizol Reagent (Life Technologies, Carlsbad, CA, USA) according to the manufacturer's protocol. Reverse transcription was performed according to the protocol of the Improm-II Reverse Transcriptase System (Promega, Madison, WI, USA). The qPCR was performed as described in the SYBR premix ExTaq instructions (Takara Biomedical Tech., Beijing, China) with an ABI Prism 7000 System (Applied Biosystems, Bedford, MA, USA). The forward and reverse sequences of primer were used for RT-qPCR, which were synthesized by Shanghai Biotechnology Co., Ltd. as following:

(I) GATA-1: F. ACGACCACTACAACACTCTGGC; $R$. TTGCGGTTCCTCGTCTGGATTC;

(II) GATA-2: F. CAGCAAGGCTCGTTCCTGTTCA; R. ATGAGTGGTCGGTTCTGCCCAT;

(III) G A P D f or normalization: $F$. $G A A G G T G A A G G T C G G A G T C ; R$. GAAGATGGTGATGGGATTTC.

\section{Western Blot analysis for GATA proteins and protein kinases}

Total cell lysate or nuclear protein sample at $20 \mu \mathrm{g}$ per lane with electrophoresis sample buffer were carried out electrophoresis by SDS-polyacrylamide gel electrophoresis (PAGE) gel. The proteins were transferred from gel to nitrocellulose membrane (Amersham, USA) using an electroblotting apparatus (Bio Rad, Hercules, CA, USA). After blocking, the membranes of nuclear protein were incubated in $0.5 \mu \mathrm{g} / \mathrm{mL}$ of primary antibodies (Santa Cruz Biotechnology, Santa Cruz, CA, USA) against GATA-1, GATA-2, P-GATA-1, and P-GATA-2, and the membranes of total cell lysate were incubated in primary antibody (Santa Cruz, USA) against AKT-1 and MAPK14, respectively, for $1 \mathrm{~h}$, then the membranes of proteins above incubated with peroxidase conjugated secondary antibodies (Santa Cruz, USA). The special bands from conjugation reaction of protein antigen and antibody were visualized by Luminol of the enhanced chemiluminescence (ECL) kit (Santa Cruz, USA). The experiment above was repeated 3 times.

\section{EMSA and antibody Gel Supershift assay for GATA transcription factor}

As in the authors' previous study of EMSA for the DNA binding reaction $(13,14)$, each sample of $10 \mu \mathrm{g}$ nuclear protein was incubated in $25 \mu \mathrm{L}$ total reaction volume containing $20 \mathrm{mM}$ HEPES, $50 \mathrm{mM} \mathrm{NaCl}, 0.1 \mathrm{mM}$ EDTA, $1 \mathrm{mM}$ dithiothreitol (DTT), 5\% glycerol, and $2.5 \mu \mathrm{g}$ poly $\mathrm{dI} / \mathrm{dC}$ for $15 \mathrm{~min}$ at $4{ }^{\circ} \mathrm{C}$. The probe was double-stranded transcription factor of GATA consensus oligonucleotide (Santa Cruz, USA) contained binding site for GATA-1, GATA-2, and GATA-3 transcription factor: with the sequence of 5'-CAC TTG ATA ACA GAA AGT GAT AAC TCT-3'. The GATA consensus ds-oligonucleotide was radiolabeled with the polynucleotide kinase and [a-32P] ATP (Amersham, USA). We added 50,000 cpm of oligonucleotide to the reaction mixture and incubated it for $30 \mathrm{~min}$. The reaction products were analyzed by electrophoresis in $6 \%$ polyacrylamide gel with 0.25 -fold tris borate EDTA (TBE) running buffer (22.3 mM Tris, $22.2 \mathrm{mM}$ borate, and 0.5 mM EDTA). The gel was dried, and the bands of GATA-DNA binding complex were visualized by autoradiography. The above experiment was repeated 3 times.

The experiment of antibody gel supershift assay was similar to EMSA, but GATA-1, GATA-2 and GATA-3 antibodies were added to the reaction mixture, before the probe binding to the nucleoprotein. Then, reaction products were analyzed by electrophoresis in $6 \%$ polyacrylamide gel. The gel was dried, and the bands of GATA-DNA binding complex were visualized by autoradiography. The above experiment was repeated 3 times.

\section{Statistical analysis}

Data were presented as the mean \pm standard deviation. Student's $t$-test was used to analyze experimental data containing two groups, and one-way analysis of variance (ANOVA) was used to analyze experimental data with $\geq 3$ groups. Statistical analysis was performed using SPSS version 16.0 (IBM Corp., Armonk, NY, USA), A $\mathrm{P}$ value $<0.05$ was considered to indicate a statistically significant difference.

\section{Results}

\section{The morphology of colony formation of hematopoietic progenitor cells}

The colony formation morphology of CFU-GM, CFU-E, and CFU-MK derived from mouse bone marrow in agar semisolid culture is shown in Figure 1. The CFUGM colony contained more than 40 cells stained by 


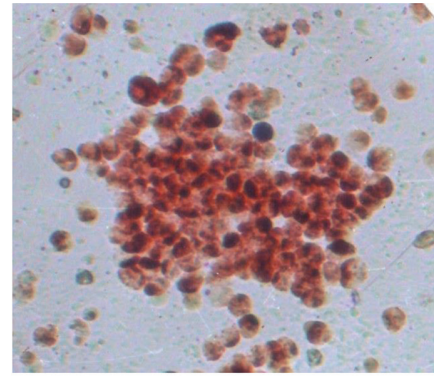

CFU-GM colony

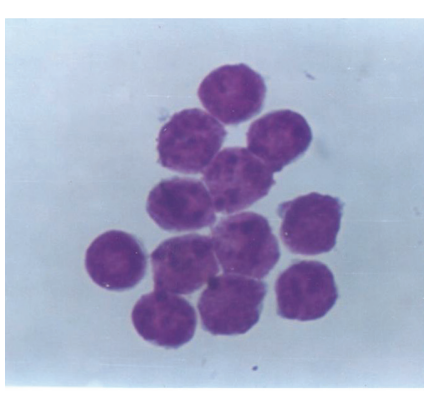

CFU-E colony

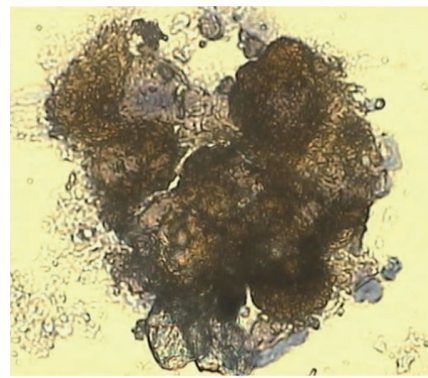

CFU-MK colony

Figure 1 The morphology of colony formation in semisolid culture of mouse bone marrow in situ. CFU-GM colony stained by HE ( $\times 200$ ); CFU-E colony by Wright staining $(\times 400)$; CFU-MK colony identified by acetylcholinesterase staining $(\times 400)$.

dimethoxybenzidine after semisolid culture of bone marrow in situ $(\times 200)$; CFU-E colony contained more than 8 cells by Wright's staining in situ $(\times 400)$; and CFUMK colony contained more than 4 cells identified by acetylcholinesterase staining in situ $(\times 400)$. The colony forming assay represented a colony derived from a hematopoietic progenitor cell, the hematopoietic cells within CFU-GM or CFU-E colonies referred to granulocytic or erythroid precursor and immature cells respectively, and the cells within CFU-MK colonies referred to megakaryocytic precursor and immature cells.

\section{PDS-C promoted the proliferation of hematopoietic progenitor cells in normal mice}

Table 1 showed that CFU-GM and CFU-E colony formation in response to PDS-C at $10,25,50 \mathrm{mg} / \mathrm{L}$ was enhanced compared to those of untreated controls, respectively (all $\mathrm{P}<0.01$ ) in bone marrow culture of normal mice, and PDS-C increased the colony numbers by $28.5 \% \pm 3.4 \%$ to $42.2 \% \pm 4.6 \%, 26.5 \% \pm 3.2 \%$ to $42.4 \% \pm 4.5 \%$ respectively, which were significant more than those of untreated controls. Also CFU-MK colony formation of bone marrow in the presence of PDS-C at $10,25,50 \mathrm{mg} / \mathrm{L}$ was elevated compared to without PDS-C control, respectively $(\mathrm{P}<0.01)$, and PDS-C increased colony numbers by $25.7 \% \pm 3.1 \%$ to $40.9 \% \pm 4.3 \%$, which were significant more than untreated control. The results above suggest that PDS-C can effectively promote proliferation of granulocytic, erythroid and megakaryocytic hematopoietic progenitor cells of mouse bone marrow in a dose-dependent manner.

The positive control of Testosterone $10^{-7} \mathrm{M}$ were effective to promote proliferation of both erythroid and megakaryocytic progenitor cells in normal mice, and increased the CFU-E, CFU-MK colony numbers by $45.1 \% \pm 4.6 \%, 24.3 \% \pm 2.6 \%$ respectively, while, granulocytic hematopoietic progenitor cells were not response to Testosterone, the colony numbers were no significant difference between Testosterone treated and untreated control group.

\section{PDS-C promoted the proliferation of hematopoietic progenitor cells in AA mice}

Table 2 showed that CFU-GM, CFU-E colony formation of AA mouse bone marrow in response to PDS-C at $10,25,50 \mathrm{mg} / \mathrm{L}$ was enhanced compared to those of untreated controls, respectively (all $\mathrm{P}<0.01$ ), and PDS-C increased colony numbers by $32.5 \% \pm 4.9 \%$ to $52.1 \% \pm 7.3 \%$, $31.1 \% \pm 4.3 \%$ to $53.1 \% \pm 7.4 \%$, which were more than those of untreated controls. Also CFU-MK colony formation in response to PDS-C at 10, 25, $50 \mathrm{mg} / \mathrm{L}$ was elevated compared to without $\mathrm{PDS}-\mathrm{C}$ control, respectively (all $\mathrm{P}<0.01$ ), and PDS-C increased colony numbers by $29.7 \% \pm 3.7 \%$ to $53.2 \% \pm 7.1 \%$, which were more than untreated control. The results above suggest that PDS-C is an effective component not only to promote proliferation of myeloid, erythroid and megakaryocytic hematopoietic progenitor cells in normal mice, but also to enhance hematopoiesis for AA mice when are in a state of myelosuppression and hypohematopoietic function.

The positive control of Testosterone $10^{-7} \mathrm{M}$ were effective to promote proliferation of both erythroid and megakaryocytic progenitor cells in AA mice, and increased the CFU-E, CFU-MK colony numbers by $58.3 \% \pm 7.7 \%$, $28.1 \% \pm 3.5 \%$ respectively, while, granulocytic hematopoietic progenitor cells were not response to Testosterone, the colony numbers were no significant difference between 
Table 1 PDS-C increased the colony formation of granulocytic, erythroid, and megakaryocytic progenitor cells in normal mice $(\bar{x} \pm s, \mathrm{n}=8)$

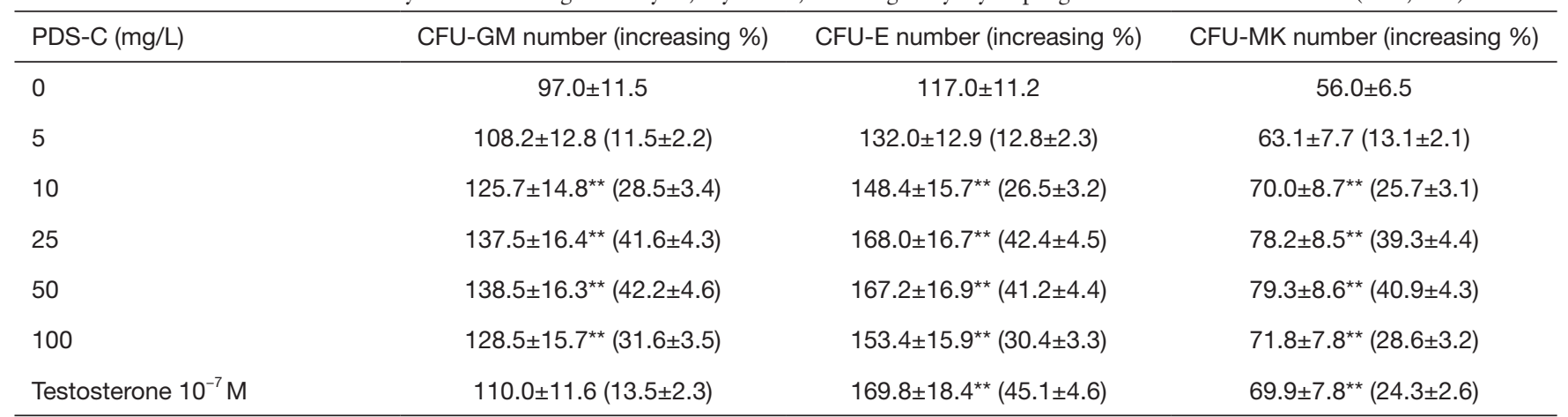

**, $\mathrm{P}<0.01$, vs. untreated control cells. PDS-C, panaxadiol saponins component; CFU-GM, colony formation unit granulocyte and macrophage; CFU-E, colony formation unit-erythroid; CFU-MK, colony formation unit megakaryocytic progenitor.

Table 2 PDS-C increased the colony formation of granulocytic, erythroid and megakaryocytic progenitor cells in AA mice $(\bar{x} \pm s, \mathrm{n}=8)$

\begin{tabular}{|c|c|c|c|}
\hline PDS-C (mg/L) & CFU-GM number (increasing \%) & CFU-E number (increasing \%) & CFU-MK number (increasing \%) \\
\hline 5 & $35.1 \pm 3.6(13.1 \pm 3.4)$ & $44.7 \pm 8.7(13.0 \pm 1.9)$ & $29.4 \pm 4.2(12.1 \pm 2.0)$ \\
\hline 10 & $41.1 \pm 3.8^{\star \star}(32.5 \pm 4.9)$ & $52.4 \pm 5.3^{* *}(31.1 \pm 4.3)$ & $34.1 \pm 4.3^{\star *}(29.7 \pm 3.7)$ \\
\hline 25 & $46.5 \pm 4.1^{\star \star}(49.2 \pm 6.1)$ & $60.3 \pm 6.1^{* *}(51.6 \pm 6.7)$ & $39.6 \pm 5.2^{\star \star}(50.4 \pm 6.1)$ \\
\hline 100 & $42.3 \pm 4.0^{\star \star}(34.9 \pm 1.6)$ & $54.0 \pm 6.0^{\star \star}(35.1 \pm 5.3)$ & $35.1 \pm 4.9^{\star \star}(34.2 \pm 7.1)$ \\
\hline Testosterone $10^{-7} \mathrm{M}$ & $36.0 \pm 3.7(15.1 \pm 3.5)$ & $63.0 \pm 6.1^{\star *}(58.3 \pm 7.7)$ & $33.7 \pm 4.2^{\star \star}(28.1 \pm 3.5)$ \\
\hline
\end{tabular}

**, $\mathrm{P}<0.01$, vs. untreated control cells. PDS-C, panaxadiol saponins component; CFU-GM, colony formation unit granulocyte and macrophage; CFU-E, colony formation unit erythroid; CFU-MK, colony formation unit megakaryocytic progenitor.

Testosterone treated and untreated control group.

\section{PDS-C up-regulated genes expression related to proliferation and differentiation}

Mouse bone marrow cultures of CFU-GM, CFU-E and CFU-MK were treated by PDS-C for 3 days, and the granulocytic, erythroid and megakaryocytic hematopoietic cells (precursors/immature cells) were harvested after culture for gene microarray analysis (Figure 2). The results of gene microarray showed PDS-C up-regulated genes by more than 3 times in 3 lineages of hematopoietic cells, which could be classified into 11 categories according to their functions, including methyl and acetyl transferase, differentiation related, and inhibiting apoptosis and transcriptional regulatory protein, cell cycle proteins, signaling mediated protein kinase, and receptor related, DNA and RNA polymerase, phosphatase, carrier and transporters, etc.

It was shown that PDS-C induced up-regulation of 66 genes by more than 3 times in granulocytic hematopoietic cells, mainly including signaling mediated protein kinases, transcriptional regulatory proteins, differentiationrelated, and cell cyclins. Additionally, PDS-C induced upregulation of 62 genes by more than 3 times in erythroid hematopoietic cells, mainly including signaling mediated proteins kinases, phosphatases, transcriptional factor, receptor-related proteins. In megakaryocytic hematopoietic cells, PDS-C up-regulated 128 genes by more than three 3 times, mainly including signaling mediated proteins kinases, phosphatases, transcriptional factor, methyl and acetyltransferases, and receptor-related genes. The coexpression levels of 12 genes were increased by more than 3 times in 3 lineages of hematopoietic cells, mainly including methylated and acetylated transferases, signaling mediated protein kinases, and phosphatases. 


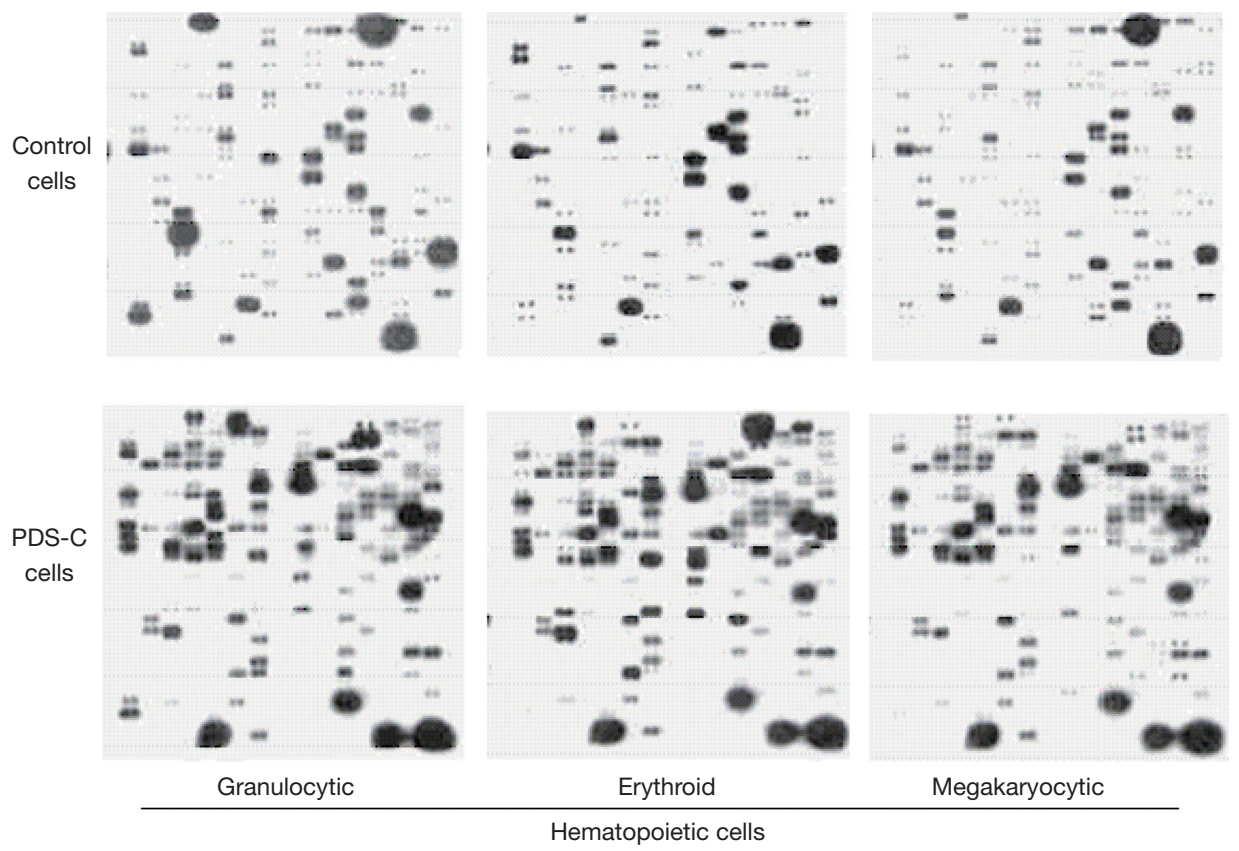

Figure 2 PDS-C induced gene profile in three lineages of hematopoietic cells. PDS-C, panaxadiol saponins component.

The microarray results showed that PDS-C up-regulated genes of GATA transcription factors and protein kinases AKT-1 and MAPK14, as shown in Table 3. The genes of GATA-1 and GATA-2 were up-regulated in PDS-C treated erythroid and megakaryocytic hematopoietic cells. Meanwhile, the genes of $A K T-1$ and MAPK14 protein kinases as upstream activation protein of GATA-1 and GATA-2 were also up-regulated by PDS-C treatment, which were in the relationship with proliferation and differentiation in hematopoietic cells.

\section{PDS-C up-regulated the mRNA expression of GATA-1 and GATA-2}

The mRNA expression levels of GATA-1 and GATA2 transcription factors related to proliferation and differentiation were analyzed by RT-qPCR, so as to confirm the results of gene microarray results. Table 4 shows that the mRNA expression levels of GATA-1 and GATA-2 genes in PDS-C treated erythroid and megakaryocytic hematopoietic cells were significantly higher than those of untreated control cells (all $\mathrm{P}<0.01$ ). The results of $\mathrm{RT}-\mathrm{qPCR}$ were consistent with their gene microarray profile, suggesting the efficacy of PDS-C on enhancing proliferation and differentiation in erythroid and megakaryocytic hematopoietic cells by means of up-regulation of GATA-1 and GATA-2 transcription factors.

\section{PDS-C enhanced the expression level and phosphorylation status of GATA protein}

Western blot results showed that GATA-1 and GATA2 proteins were at low expression level in the nuclei of untreated erythroid and megakaryocytic hematopoietic cells (lane 3,5), while, PDS-C induced up-regulation of GATA-1 and GATA-2 proteins, and significantly increased the density of specific bands (lane 4, 6), which was consistent with the results of gene expression profile induced by PDS-C (Figure 3). However, there were no obvious alterations for both GATA-1 and GATA-2 proteins in PDS-C treated granulocytic cells (lane 2) compared with untreated control cells (lane 1).

Phosphorylation status of GATA-1 and GATA-2 proteins were at low degree in the nuclei of untreated erythroid and megakaryocytic hematopoietic cells (lane $3,5)$ by using phosphorylated antibody of P-GATA-1 and P-GATA-2 (Figure 3). Meanwhile, PDS-C effectively enhanced phosphorylated degree of GATA-1 and GATA2 proteins (lane 4,6 ), and significantly increased the specific band density of both P-GATA-1 and P-GATA-2 protein in comparison with untreated control cells (lane $3,5)$. This indicates that PDS-C can not only up-regulate 
Table 3 PDS-C up-regulated genes of transcription factors and protein kinases analyzed with gene microarray in hematopoietic cells

\begin{tabular}{lccc}
\hline Gene name & Granulocyte (fold) & Erythrocyte (fold) & Megakaryocyte (fold) \\
\hline GATA-1 & - & 3.8 & 4.1 \\
GATA-2 & - & 5.9 & 4.7 \\
AKT-1 & 2.3 & 4.0 & 3.2 \\
MAPK14 & 1.6 & 4.1 & 3.3 \\
\hline
\end{tabular}

PDS-C, panaxadiol saponins component.

Table 4 PDS-C up-regulated the mRNA expression levels of GATA-1 and GATA-2 genes by RT-qPCR $(\bar{x} \pm s, \mathrm{n}=3)$

\begin{tabular}{lcrr}
\hline \multirow{2}{*}{ Hematopoietic cells } & PDS-C $(\mathrm{mg} / \mathrm{L})$ & \multicolumn{2}{c}{ Relative expression level of mRNA } \\
\cline { 3 - 4 } Erythrocyte & 0 & GATA-1 & $1.00 \pm 0.29$ \\
& 50 & $1.00 \pm 0.31$ & $2.41 \pm 0.35^{\star \star}$ \\
Megakaryocyte & 0 & $1.41 \pm 0.32^{\star \star}$ & $1.00 \pm 0.23$ \\
& 50 & $1.00 \pm 0.30$ & $2.14 \pm 0.33^{\star \star}$ \\
\hline
\end{tabular}

${ }^{\star *}, \mathrm{P}<0.01$, vs. untreated control cells. PDS-C, panaxadiol saponins component; RT-qPCR, quantitative reverse transcription polymerase chain reaction.

expression levels of GATA-1 and GATA-2, but also enhance their phosphorylated state as functional activity. However, there were no obvious alterations for both P-GATA-1 and P-GATA-2 proteins after PDS-C treatment (lane 2) in granulocytic hematopoietic cells in compared with untreated control cells (lane 1).

It is also shown in Figure 3 that the band density of AKT1 and MAPK14 protein kinases was obviously increased by PDS-C treatment in total cell lysate of erythroid and megakaryocytic hematopoietic cells (lane 4, 6) in comparison with untreated control cells (lane 3, 5), AKT-1, MAPK14 proteins were as the upstream signaling molecule for activation GATA-1, GATA-2 transcription factors. However, there were no obvious alterations for both AKT-1 and MAPK14 proteins after PDS-C treatment in granulocytic hematopoietic cells (lane 2) in comparison with untreated control cells (lane 1).

\section{PDS-C promoted DNA binding activity of GATA transcription factor}

The EMSA analysis of Figure $4 A$ shows the specific bands of GATA-DNA biding complex (lane 3-6). The band density of GATA-DNA biding complex in the nuclei of erythroid and megakaryocytic hematopoietic cells was obviously increased by PDS-C (lane 4, 6) compared with untreated control cells (lane 3, 5), suggesting that PDS-C was effective to enhance binding activity of GATA transcription factor to DNA sequence of gene regulatory region. However, there was no obvious change in granulocytic hematopoietic cells with and without PDS-C treatment (lane 2,1).

In order to realize the main component of GATA-DNA binding complex initiated by PDS-C, the nuclei proteins of PDS-C treated erythroid and megakaryocytic hematopoietic cells (lane 1,5) were used for performing antibody gel supershift assay, by adding 3 kinds of antibodies against GATA-1 (lane 2, 6), GATA-2 (lane 3, 7), and GATA-3 (lane 4, 8), respectively, as shown in Figure $4 B$. The specific band density of GATA-DNA complex was obviously decreased in the presence of GATA-2 antibody, indicating that the main components of GATA-DNA complex as antigen could combine with GATA-2 antibody in both cells respectively, so as to increase their molecular weight, causing the bands move up (lane 3 and 7, the arrow points to the moving up bands) or almost disappear. The specific band density of GATA-DNA complex of megakaryocytic hematopoietic cells was partially reduced in the presence of GATA1 antibody (lane 6). However, there were no obvious alterations for GATA-DNA complex in the presence of GATA-3 antibody in both hematopoietic cells (lane 4, 8). 

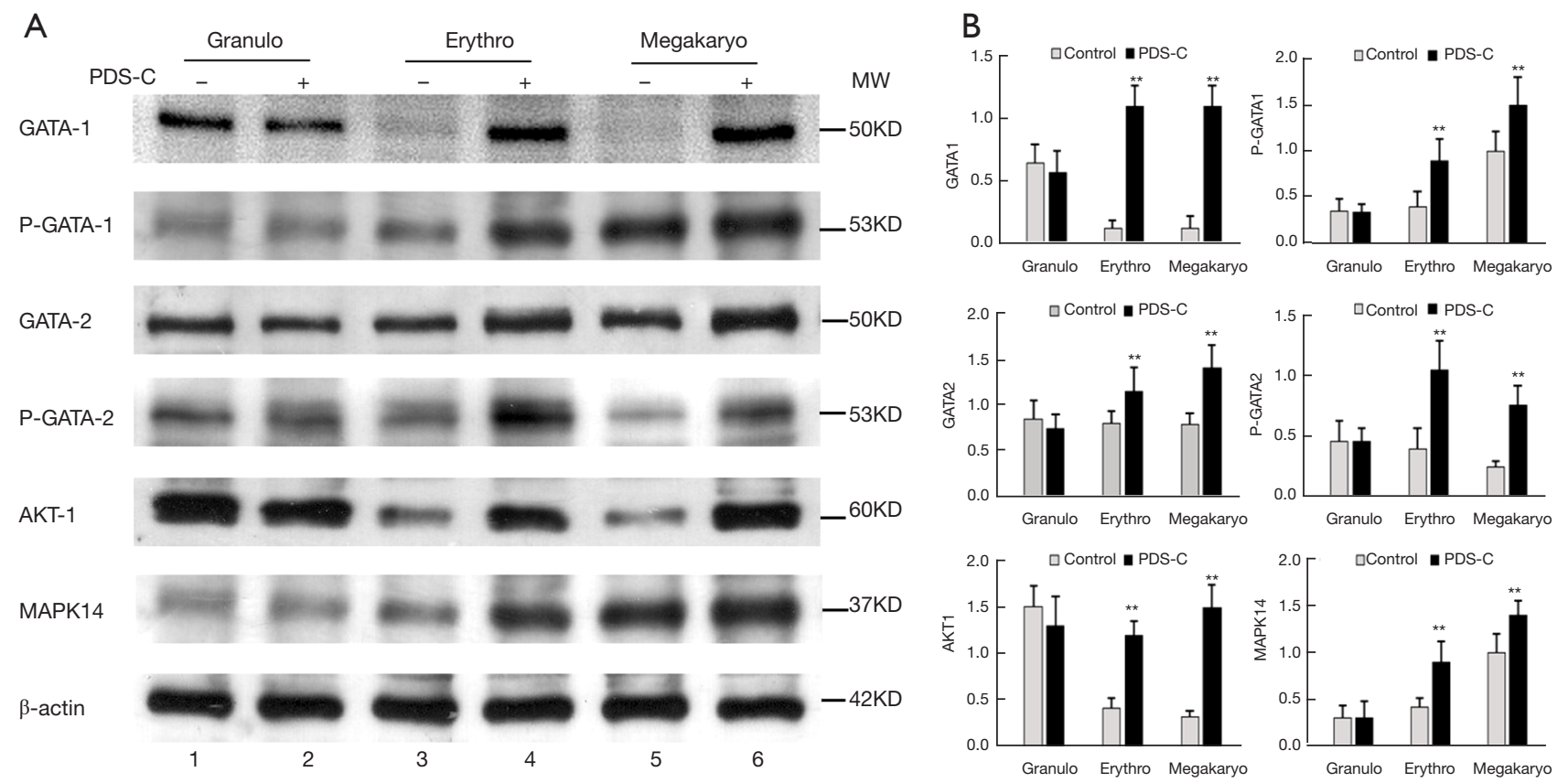

Figure 3 PDS-C up-regulated expression level and phosphorylated status of GATA protein by Western Blot. Notes: 2, 4, 6: PDS-C treated granulocytic, erythroid and megakaryocytic hematopoietic cells respectively; $1,3,5$ : the untreated control cells. ${ }^{* *}, \mathrm{P}<0.01$, vs. untreated control cells. PDS-C, panaxadiol saponins component.

These results suggest that the main component of GATADNA biding complex is mediated by PDS-C is GATA-2 transcription factor in both cells, and followed by GATA-1 protein only in megakaryocytic cells.

\section{Discussion}

The Chinese ginseng herb is praised as the first effective medicine for invigorating qi, which can replenish qi and nourish blood, tranquilize the mind, increase intelligence, and invigorate qi to assist yang. The main active components of ginseng are ginsenosides, but so far, ginsenosides cannot be synthesized artificially base on the existing technology and conditions. In order to find and determine the effective component within total saponins of ginsenosides for treating hemocytopenia, we extracted and isolated multiple components from Chinese ginseng herb, then, analyzed various components for their biological activities of hematopoiesis and found a biological active PDS-C. Our research team has investigated and developed a new Chinese patent medicine named Pai-neng-da (PDS-C) capsules for the clinical need, the project of clinical research and industrialization is being supported by National Major
Project for the Innovative New Drugs of "the 13th FiveYear Plan".

Our previous experiment demonstrated that PDS-C was effective, not only to prompt proliferation of human hematopoietic cells and increase the colony formation of CFU-GM, CFU-E, and CFU-MK, but also to induce differentiation of human $\mathrm{CD}_{3} 4^{+}$stem/ progenitor hematopoietic cells and CHRF-288 and Meg01 megakaryoblasts in vitro (8). Treatment with PDS-C improved myelosuppression and hemocytopenia in AA mouse models and increased the peripheral blood counts though promotion proliferation of hematopoietic progenitors (15). We also reported a cohort study of 36 patients with chronic AA who received Pai-neng-da Capsule orally at a dose of $240 \mathrm{mg} / \mathrm{d}$ in combination with andriol (at a reduced dose) or CsA for 6 months. The results indicate that Pai-neng-da in combination with andriol or CsA is effective to increase the peripheral blood counts without causing side effects, and allow dose reduction of andriol, thus minimizing the side effects of andriol (16).

In this study, the mouse bone marrow culture showed that PDS-C at 10,25 , and $50 \mathrm{mg} / \mathrm{L}$ was effective to increase colony numbers of CFU-GM, CFU-E, and CFU-MK 


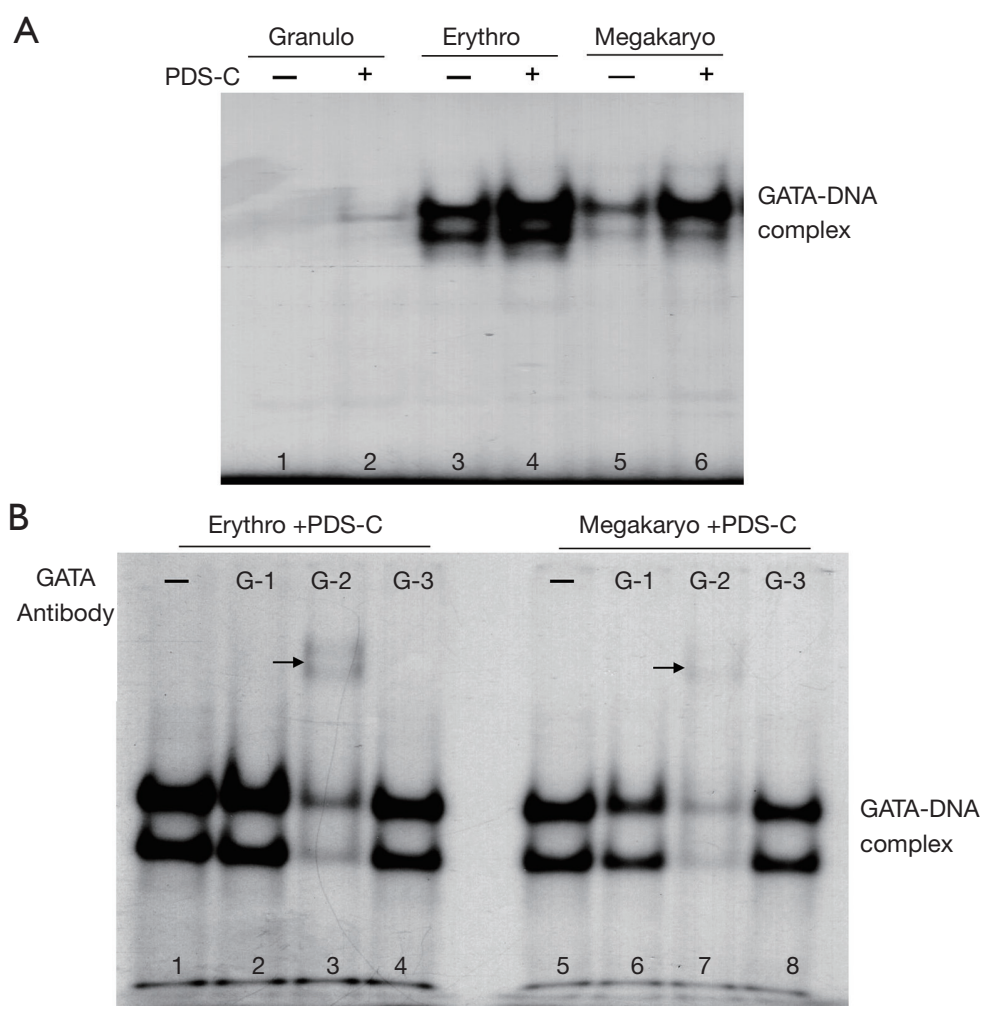

Figure 4 PDS-C enhanced DNA binding activity of GATA transcription factor in mouse hematopoietic cells. (A) PDS-C induced GATADNA complex detected by EMSA; (B) the main components of GATA-DNA complex analyzed by antibody gel supershift assay using GATA-1, 2, and 3 antibodies respectively. The main components of GATA-DNA complex could combine with GATA-2 antibody in both cells respectively, so as to increase their molecular weight, causing the bands move up, the arrow points to the moving up bands. PDS-C, panaxadiol saponins component.

in normal mice, as well to enhance colony formation of 3 lineages hematopoietic cells in AA mice compared with the untreated control in a dose-dependent manner. These results suggest that PDS-C has efficacy in promoting proliferation of granulocytic, erythroid, and megakaryocytic hematopoietic progenitor cells, not only for normal mice, but also for AA mice during the period of myelosuppression and hypohematopoietic function, implying the efficacy of PDS-C may involve growth factor-like action and synergistic effects with growth factors $(7-9,16)$. The positive control of Testosterone $10^{-7} \mathrm{M}$ also were effective to promote proliferation of both erythroid and megakaryocytic progenitor cells in normal or AA mice, and increased the CFU-E, CFU-MK colony numbers respectively. While, granulocytic hematopoietic progenitor cells were not response to Testosterone, the colony numbers were no significant increased.

Since the action mechanism of PDS-C on promoting hematopoiesis is not yet clear, in this study, gene microarray analysis provided evidence of gene expression profiles for the role of PDS-C. The genes that PDS-C up-regulated by more than 3 times were mainly associated with proliferation and differentiation of hematopoietic cells, which could be classified into 11 categories according to their functions. The gene expression profile initiated by PDS-C also provided the up-regulated genes, including GATA-1 and GATA-2 transcription factors to different degree in PDS-C treated erythroid and megakaryocytic cells. In order to confirm the results of gene membrane microarray, we analyzed the mRNA expression levels of GATA-1 and GATA-2 in PDS-C treated erythroid and megakaryocytic cells. The RT-qPCR results showed that PDS-C significantly increased mRNA expression of GATA-1 and GATA-2 genes, which was consistent with the gene microarray profile. It suggests that the efficacy of PDS-C on enhancing proliferation and differentiation of hematopoietic cells 
involves in GATA-1 and GATA-2 transcription factor of intracellular signal pathway.

Western blot results showed that PDS-C induced upregulation of GATA-1 and GATA-2 protein in the nuclei of erythroid and megakaryocytic hematopoietic cells, which was consistent with the results of gene expression profile and RT-qPCR after treatment with PDS-C. Furthermore, PDS-C effectively enhanced phosphorylated status of GATA-1 and GATA-2 proteins, both P-GATA-1 and P-GATA-2 proteins were increased in comparison with untreated control cells. This indicates that PDS-C can not only up-regulate expression levels of GATA-1 and GATA-2 proteins, but also enhance their phosphorylated functional activity state.

The DNA binding activity of GATA protein was enhanced obviously in PDS-C treated erythroid and megakaryocytic hematopoietic cells. This suggests that PDS-C is effective for the GATA transcription factor family in increasing their expression, translocating from cytoplasm into the nucleus, and accentuating binding activities to DNA. The main composition of GATA-DNA complex initiated by PDS-C was GATA-2 transcription factors in both cells, and followed by GATA-1 protein only in megakaryocytic cells, the GATA-DNA complex as the antigen protein can combine with GATA-2 and GATA1 antibody, respectively. The GATA transcription factor belongs to the family of zinc finger structure, and the regulatory sequence T/A (GATA) $\mathrm{G}$ is the binding site on the promoter and/or enhancer of the target gene. A total of 6 GATA transcription factors have been discovered, but those closely related to hematopoiesis are GATA-1, GATA-2, and GATA-3, their main functions are to mediate the response to growth factors, regulate the expression of target genes, and play an important regulatory role in cell proliferation and differentiation (17-21). Among them, GATA-1 is involved in the development of erythrocyte and differentiation of megakaryocyte $(17,18)$. Hematopoietic development requires the transcription factor GATA-2, the genes regulated by GATA-2 are involved in the proliferation of early hematopoietic cells, and GATA-2 mutations cause diverse pathologies, whereas $(19,20)$. GATA-3 is closely related to the differentiation and development for T cells (21). Our results display that PDS-C plays a role in the promotion and proliferation of erythroid and megakaryocytic hematopoietic progenitor cells by means of GATA-2 transcription factor, and induces differentiation of megakaryocytic hematopoietic progenitor cells though GATA-2 transcription factor.
The intracellular signaling pathway acts as a bridge between the cell surface and the target gene in the nucleus, and plays an important role in the response to external stimuli. As the upstream signaling molecule for activation of GATA-1 and GATA-2 transcription factors, AKT-1 and MAPK14 protein kinases were obviously increased by PDS-C treatment in total cell lysate of erythroid and megakaryocytic cells. The function of AKT1 is phosphorylated and activated by GATA-1 transcription factor (22), while, the role of MAPK14 is phosphorylated and activated by GATA-2 transcription factor (23).

\section{Conclusions}

It may be that PDS-C plays the role of mediating and activating the signaling pathway of the GATA transcription factor, by virtue of up-regulating their mRNA expression and protein synthesis, enhancing phosphorylation extent and DNA binding activity, as well as up-regulating AKT-1 and MAPK14 protein kinases, thereby regulating the proliferation and differentiation of related target genes, resulting in the promotion of hematopoiesis. These results preliminarily reveal the action mechanism of PDS-C, but a more comprehensive mechanism remains to be elucidated through future research.

\section{Acknowledgments}

The authors are thankful for the guidance and support of the Animal Center of Zhejiang Chinese Medical University. Funding: This study was supported by the National Natural Science Foundation of China (No. 81774068), National Major Project for the Innovative New Drugs of "the 13th Five-Year Plan" (No. 2016ZX09101071), National Natural Science Foundation of China (Youth Project, No. 81703907), Project of Administration of Traditional Chinese Medicine of Zhejiang Province (No. 2019ZA058). Authors declared that the funding source did not participate in the study design; collection, analysis and interpretation of data; in the writing of the manuscript; and in the decision to submit the manuscript for publication.

\section{Footnote}

Reporting Checklist: The authors have completed the ARRIVE reporting checklist. Available at https://atm. amegroups.com/article/view/10.21037/atm-21-4800/rc 
Data Sharing Statement: Available at https://atm.amegroups. com/article/view/10.21037/atm-21-4800/dss

Conflicts of Interest: All authors have completed the ICMJE uniform disclosure form (available at https://atm. amegroups.com/article/view/10.21037/atm-21-4800/coif). The authors have no conflicts of interest to declare.

Ethical Statement: The authors are accountable for all aspects of the work in ensuring that questions related to the accuracy or integrity of any part of the work are appropriately investigated and resolved. Animals were cared for in accordance with the guidelines of the National Science and Technology Committee of China. All procedures and animal experiments were approved by the Animal Care and Use Committee of the Animal Center of Zhejiang Chinese Medical University (No. ZSLL-2015-126).

Open Access Statement: This is an Open Access article distributed in accordance with the Creative Commons Attribution-NonCommercial-NoDerivs 4.0 International License (CC BY-NC-ND 4.0), which permits the noncommercial replication and distribution of the article with the strict proviso that no changes or edits are made and the original work is properly cited (including links to both the formal publication through the relevant DOI and the license). See: https://creativecommons.org/licenses/by-nc-nd/4.0/.

\section{References}

1. Dezern AE, Brodsky RA. Clinical management of aplastic anemia. Expert Rev Hematol 2011;4:221-30.

2. Garg R, Faderl S, Garcia-Manero G, et al. Phase II study of rabbit anti-thymocyte globulin, cyclosporine and granulocyte colony-stimulating factor in patients with aplastic anemia and myelodysplastic syndrome. Leukemia 2009;23:1297-302.

3. Ma FS, Qian XD, Gao RL. Efficacy observation of Shengxue-ling capsules (total saponins of panax ginseng) in treating chronic aplastic anemia. J Zhejiang Clin Med (Chin) 2004;6:952-3.

4. Gao RL, Xu CL, Jin JM, et al. Effect of total saponins of Panax ginseng on hematopoietic progenitor cells in normal human and aplastic anemia patients. Chin J Integr Trad West Med (Chin) 1992;12:285-7, 261-2.

5. Chen XH, Gao RL, Xu WH, et al. Effect of ginsenosides in inducing proliferation and transcription factor of erythrocytic, granulo-monocytic and megakarocytic cell lines. Zhongguo Zhong Xi Yi Jie He Za Zhi 2001;21:40-2.

6. Gao RL. Research and development of new Chinese materia medica for treatment of refractory hematopathy by establishment and application of multiple technique platforms. Chin J Integr Med 2007;13:95-7.

7. Gao RL, Chong BH. Research and development of the effective components of panaxdiol saponin as new Chinese patent medicine for treating hemocytopenia. Chin J Integr Med 2012;18:897-902.

8. Wen WW, Sun X, Zhuang HF, et al. Effects of panaxadiol saponins component as a new Chinese patent medicine on proliferation, differentiation and corresponding gene expression profile of megakaryocytes. Chin J Integr Med 2016;22:28-35.

9. Lin X, Yin L, Gao R, et al. The effects of panaxadiol saponins on megakaryocytic maturation and immune function in a mouse model of immune thrombocytopenia. Exp Hematol 2015;43:364-73.

10. Yin LM, Jiang HF, Wang X, et al. Effects of sodium copper chlorophyllin on mesenchymal stem cell function in aplastic anemia mice. Chin J Integr Med 2013;19:360-6.

11. Jin J, Tao H, Gao RL. Effect of ginsenosides on proliferation and differentiation of human CD34+ hematopoietic stem/progenitor cells. Zhongguo Zhong Xi Yi Jie He Za Zhi 2000;20:673-6.

12. Fang GL, Gao RL, Lin XJ, et al. Effects of Ginseng panaxadiol saponins on proliferation and differentiation of human bone marrow CD34+ cells. Zhongguo Shi Yan Xue Ye Xue Za Zhi 2007;15:776-9.

13. Gao RL, Chen XH, Lin XJ, et al. Effects of notoginosides on proliferation and upregulation of GR nuclear transcription factor in hematopoietic cells. Acta Pharmacol Sin 2007;28:703-11.

14. Sun X, Gao RL, Lin XJ, et al. Panax notoginseng saponins induced up-regulation, phosphorylation and binding activity of MEK, ERK, AKT, PI-3K protein kinases and GATA transcription factors in hematopoietic cells. Chin J Integr Med 2013;19:112-8.

15. Zhang AP, Gao RL, Yin LM, et al. Panoxadiol saponins Induce upregulation of MAPK/ERK signal pathway in bone marrow cells of aplastic anemia mice. Chin J Pathophysi (Chin) 2018;34:686-92.

16. Kuang YM, Zhu Y, Gao RL, et al. Clinical study of paineng-da capsule in the treatment of chronic aplastic anemia. Chin J Integr Med 2016;22:124-9.

17. Lally J, Boasman K, Brown L, et al. GATA-1: A potential novel biomarker for the differentiation of essential thrombocythemia and myelofibrosis. J Thromb Haemost 
2019;17:896-900.

18. Dalby A, Ballester-Beltrán J, Lincetto C, et al. Transcription Factor Levels after Forward Programming of Human Pluripotent Stem Cells with GATA1, FLI1, and TAL1 Determine Megakaryocyte versus Erythroid Cell Fate Decision. Stem Cell Reports 2018;11:1462-78.

19. Mehta C, Johnson KD, Gao X, et al. Integrating Enhancer Mechanisms to Establish a Hierarchical Blood Development Program. Cell Rep 2017;20:2966-79.

20. Hewitt KJ, Katsumura KR, Matson DR, et al. GATA Factor-Regulated Samd14 Enhancer Confers Red Blood Cell Regeneration and Survival in Severe Anemia. Dev Cell 2017;42:213-225.e4.

21. Tindemans I, Serafini N, Di Santo JP, et al. GATA-

Cite this article as: Dai TY, Lan JJ, Gao RL, Zhao YN, Yu XL, Liang SX, Liu WB, Sun X. Panaxdiol saponins component promotes hematopoiesis by regulating GATA transcription factors of intracellular signaling pathway in mouse bone marrow. Ann Transl Med 2022;10(2):38. doi: 10.21037/atm-214800
3 function in innate and adaptive immunity. Immunity 2014;41:191-206.

22. Garcia-Rodriguez S, Callejas-Rubio JL, OrtegoCenteno N, et al. Altered AKT1 and MAPK1 gene expression on peripheral blood mononuclear cells and correlation with T-helper-transcription factors in systemic lupus erythematosus patients. Mediators Inflamm 2012;2012:495934.

23. Cheng F, Twardowski L, Fehr S, et al. Selective p38a MAP kinase/MAPK14 inhibition in enzymatically modified LDL-stimulated human monocytes: implications for atherosclerosis. FASEB J 2019;27:674-86.

(English Language Editor: J. Jones) 